\section{Surgeons develop composite bone transplants}

Deborah Josefson, San Francisco

Composite bone transplants using cadaveric and host bone may become a standard method of repairing bony defects. A 6 year old boy, Adam Johnson, underwent this pioneering procedure nine months ago and has shown good results.

Adam lost most of his humerus to cancer and faced the choice of further amputation and a prosthesis or the experimental procedure. Orthopaedists at Joe DiMaggio's Children's Hospital and at Shands Hospital in Gainesville, Florida, performed the surgery, in which they fused the humerus from a cadaver to a portion of Adam's fibula and transplanted it to the area of his humerus.

Although cadaveric bone transplants are not new, fusing them to a live bone is. The fusion allows continued bony growth as the host's vascular supply and cartilaginous cap are preserved. This is especially important in children. If a cadaveric bone alone or a ceramic composite were trans- planted, the patient's arm would not achieve adult dimensions.

Commenting on the case, Michael Jofe, a member of the transplant team said: "A recent test has shown that the growth cartilage and blood supply to the transplanted fibula remain intact. There's already been evidence that the bone is growing."

In the United States about

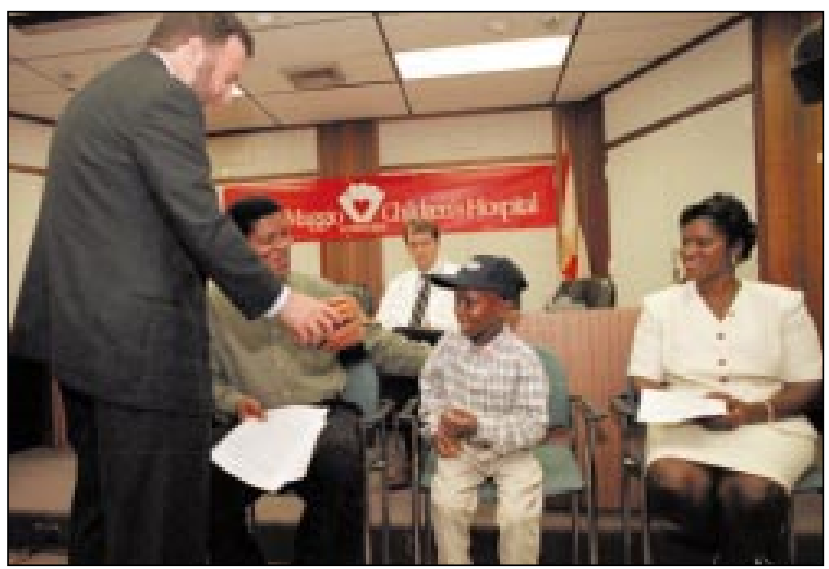

Pioneering bone surgery meant that Adam Johnson, aged 6, avoided amputation of his arm
250000 people a year undergo orthopaedic allotransplants, but only a small number of these are cadaveric. In Asia about 60 cadaveric bone transplants were performed in 1993.

The cadaveric bone is typically tested for infectious diseases and sterilised by gamma irradiation. It may be further prepared by demineralisation and by drilling laser holes in it. The holes are thought to help incorporate the bone into the host. Unlike patients receiving organ transplants, those receiving cadaveric bone transplants do not need immunosuppressive drugs. \section{$\square$}

said that, after extensive consultations with the profession, BUPA had drawn up evidence based guidelines on when a hysterectomy was necessary and therefore eligible for cover.

It had also devised an information sheet for patients, explaining the risks of and alternatives to surgery. The association would be sending these sheets to GP surgeries for distribution.

"Our aim is to provide appropriate care: the right treatment in the right place at the right time," Dr VallanceOwen said. "The Royal College of Obstetricians and Gynaecologists, in their guidelines on the management of menorrhagia, point out that one in five women will have a hysterectomy before the age of 60 .

"In at least half of these cases, menorrhagia is the main presenting problem, and in about half of those cases, a normal uterus is removed. When a gynaecologist wants to do a hysterectomy on one of our members, he or she will have to fill out a form giving the details. We are asking them to fax us the forms before the treatment for us to approve. Anything to do with carcinoma will be turned round immediately.

"If there is any question in our mind as to whether the operation is necessary, we will ask the gynaecologist to have a direct conversation with one of our gynaecologist advisers. It may be that the gynaecologist will be able to explain why they are planning to do a hysterectomy outside the guidance." Dr Vallance-Owen said that BUPA had been following a similar procedure for some time in psychiatry and oncology. 